\title{
New Approach for Solving a Class of Doubly Singular Two-Point Boundary Value Problems Using Adomian Decomposition Method
}

\author{
Randhir Singh, Jitendra Kumar, and Gnaneshwar Nelakanti \\ Department of Mathematics, Indian Institute of Technology Kharagpur, Kharagpur 721302, India \\ Correspondence should be addressed to Randhir Singh, randhir.math@gmail.com
}

Received 13 October 2012; Revised 3 December 2012; Accepted 5 December 2012

Academic Editor: Norbert Heuer

Copyright (C) 2012 Randhir Singh et al. This is an open access article distributed under the Creative Commons Attribution License, which permits unrestricted use, distribution, and reproduction in any medium, provided the original work is properly cited.

We propose two new modified recursive schemes for solving a class of doubly singular two-point boundary value problems. These schemes are based on Adomian decomposition method (ADM) and new proposed integral operators. We use all the boundary conditions to derive an integral equation before establishing the recursive schemes for the solution components. Thus we develop recursive schemes without any undetermined coefficients while computing successive solution components, whereas several previous recursive schemes have done so. This modification also avoids solving a sequence of nonlinear algebraic or transcendental equations for the undetermined coefficients with multiple roots, which is required to complete calculation of the solution by several earlier modified recursion schemes using the ADM. The approximate solution is computed in the form of series with easily calculable components. The effectiveness of the proposed approach is tested by considering four examples and results are compared with previous known results.

\section{Introduction}

Consider the following class of doubly singular two-point boundary value problems:

$$
\left(p(t) y^{\prime}(t)\right)^{\prime}=q(t) f(t, y(t)), \quad 0<t \leq 1
$$

with boundary conditions

$$
\begin{gathered}
y(0)=a_{1}, \quad y(1)=c_{1}, \\
\text { or } y^{\prime}(0)=0, \quad a y(1)+b y^{\prime}(1)=c,
\end{gathered}
$$


where $a_{1}, c_{1}, a>0, b \geq 0$, and $c$ are any finite constants. The condition $p(0)=0$ characterizes that problem (1.1) is singular and in addition to this if $q(t)$ is allowed to be discontinuous at $t=0$, then problem (1.1) is called doubly singular Bobisud [1]. Consider problem (1.1) with the following conditions on $p(t), q(t)$, and $f(t, y(t))$.

Type 1. Dirichlet boundary conditions: $y(0)=a_{1}, y(1)=c_{1}$

$\left(E_{1}\right) p \in C[0,1], p \in C^{1}(0,1]$ with $p>0$ in $(0,1]$ and $1 / p \in L^{1}(0,1)$;

$\left(E_{2}\right) q>0$ in $(0,1]$ and $\int_{0}^{1}(1 / p)\left(\int_{x}^{1} q d s\right) d x<\infty$;

(E) (i) $f \in C([0,1] \times \mathbb{R})$; (ii) $\partial f / \partial y$ exists and continuous, $\partial f / \partial y \geq 0$ for all $0 \leq t \leq 1$ and all real $y$.

Type 2. Mixed type boundary conditions: $y^{\prime}(0)=0, a y(1)+b y^{\prime}(1)=c$

$\left(E_{1}^{\prime}\right) p \in C[0,1], p \in C^{1}(0,1]$ with $p>0$ in $(0,1]$;

$\left(E_{2}^{\prime}\right) q>0$ in $(0,1], q \in L^{1}(0,1)$ and $\int_{0}^{1}(1 / p)\left(\int_{0}^{x} q d s\right) d x<\infty$;

$\left(E_{3}^{\prime}\right)$ the same as $\left(E_{3}\right)$.

In recent years, the study of such singular boundary value problems (1.1) has attracted the attention of several researchers [2-9]. In particular, if $p(t)=1, q(t)=t^{-1 / 2}$ and $f(t, y)=y^{3 / 2}$ problem (1.1) with Type 1 boundary conditions is known as Thomas-Fermi equation. Thomas [10] and Fermi [11] independently derived a boundary value problem for determining the electrical potential in an atom. The analysis leads to the nonlinear SBVP $y^{\prime \prime}=t^{-1 / 2} y^{3 / 2}$ with boundary conditions given by $y(0)=1, y\left(b_{1}\right)=0$.

Chan and Hon [12] considered the generalized Thomas-Fermi equation: $\left(t^{b_{2}} y^{\prime}\right)^{\prime}=$ $c q(t) y^{e}, y(0)=1, y(a)=0$ with $q(t)=t^{b_{2}+d_{2}}$, where $0 \leq b_{2}<1, c>0, d_{2}>-2, e>1$, which is doubly singular problem. Problem (1.1) with $q(t)=p(t)=t^{\alpha}$ where $\alpha=2$ arises in the study of the distribution of heat sources in the human head [13] with $f(t, y)=-\delta e^{-\theta y}$, $\theta>0, \delta>0$.

There is a huge literature available on numerical methods for problem (1.1) with $q(t)=1$, but very few numerical methods are available to tackle doubly singular boundary value problems. Reddien [14] studied the linear form of problem (1.1) and derived numerical methods for $q(t) \in L^{2}[0,1]$ which is stronger assumption than $\left(E_{2}^{\prime}\right)$.

Chawla and Shivakumar [15] established the existence as well as uniqueness of solution for problem (1.1) where $q(t)=p(t)=t^{\alpha}$. The existence and uniqueness for problem (1.1) have also been discussed by Dunninger and Kurtz [16] and Bobisud [1]. Later Pandey and Verma [17] extended the results on the existence uniqueness for problem (1.1) with Types 1 and 2 boundary conditions.

\subsection{Adomian Decomposition Method (ADM)}

In this subsection, we briefly describe standard ADM for nonlinear second-order equation.

Recently, many researchers [4, 18-26] have shown interest to the study of ADM for different scientific models. Adomian [18] asserted that the ADM provides an efficient and computationally suitable method for generating approximate series solution for a large 
class of differential equations. Let us consider nonlinear second-order ordinary differential equation

$$
L y+R y+N y=g(t)
$$

where $L \equiv d^{2} / d t^{2}$ is the second-order linear derivative operator, $R$ is the linear remainder operator, $N$ represents the nonlinear term, and $g(t)$ is a source term. The above equation can be rewritten as

$$
L y=g(t)-R y-N y
$$

The inverse operator of $L$ is defined as $L^{-1}(\cdot)=\int_{0}^{t} \int_{0}^{t}(\cdot) d t d t$.

Operating the inverse linear operator $L^{-1}(\cdot)$ on both the sides of (1.4) yields

$$
y=y(0)+t y^{\prime}(0)+L^{-1} g(t)-L^{-1} R y-L^{-1} N y
$$

Next, we decompose the solution $y$ and the nonlinear function $N y$ by an infinite series as

$$
y=\sum_{n=0}^{\infty} y_{n}, \quad N y=\sum_{n=0}^{\infty} A_{n}
$$

where $A_{n}$ are Adomian polynomials that can be constructed for various classes of nonlinear functions with the formula given by Adomian and Rach [19]

$$
A_{n}=\frac{1}{n !} \frac{d^{n}}{d \lambda^{n}}\left[N\left(\sum_{k=0}^{\infty} y_{k} \lambda^{k}\right)\right]_{\lambda=0}, \quad n=0,1,2, \ldots
$$

Substituting the series (1.6) into (1.5), we obtain

$$
\sum_{n=0}^{\infty} y_{n}=y(0)+t y^{\prime}(0)+L^{-1} g(t)-L^{-1} R \sum_{n=0}^{\infty} y_{n}-L^{-1} \sum_{n=0}^{\infty} A_{n}
$$

From (1.8), the various components $y_{n}$ of the solution $y$ can be determined by using the recursive relation

$$
\begin{gathered}
y_{0}=y(0)+t y^{\prime}(0)+L^{-1} g(t), \\
y_{k+1}=-L^{-1} R y_{k}-L^{-1} A_{k}, \quad k \geq 0 .
\end{gathered}
$$

For numerical purpose, the $n$-term truncated series $\psi_{n}=\sum_{m=0}^{n-1} y_{m}$ may be used to give the approximate solution. 
The ADM has been used to solve nonlinear boundary value problems (BVPs) for ordinary differential equations by several researchers [4, 20-26]. Solving nonlinear BVP by standard ADM is always a computationally involved task as it requires the computation of undetermined coefficients $y^{\prime}(0)$ in (1.9) in a sequence of nonlinear equations which increases computational complexity.

It is important to note that the standard ADM (1.9) can not be applied directly to solve two-point boundary value problem (1.1) as the component $y_{0}$ in scheme (1.9) is not independent from undetermined coefficient. Many researchers [23-25] have proposed modified ADM to overcome the difficulty by setting undermined coefficient $y^{\prime}(0)=c$, and then it will be determined by using second boundary condition satisfying $\psi_{n}(1)=y(1)$. In this case, it requires additional computational work to solve nonlinear equation $\psi_{n}(1)=y(1)$ for $c$ and $c$ may not be uniquely determined.

Benabidallah and Cherruault [24] have considered the following boundary value problem:

$$
\begin{gathered}
y^{\prime \prime}+p y^{\prime}=f(t, y), \quad a<t<b, \\
y(a)=\alpha, \quad y(b)=\beta .
\end{gathered}
$$

The inverse operator $L^{-1}=\int_{a}^{x} \int_{b}^{x} d x d x$ was proposed, then unidentified constant $y^{\prime}(b)$ is set, as $y^{\prime}(b)=\sum_{n=0}^{\infty} c_{n}$, and each solution component $y_{n}$ is obtained by the following scheme:

$$
\begin{gathered}
y_{0}=y(a)+(x-a)\left[c_{0}+y(b) p(b)\right], \\
y_{n+1}=c_{n+1}(t-a)-\int_{a}^{t} p y_{n} d t+L^{-1} A_{n}+L^{-1} p^{\prime} y_{n}, \quad n \geq 0 .
\end{gathered}
$$

In order to determine the unidentified constants $c_{n}$, it is also required $n$-term approximate solution $\psi_{n}=\sum_{m=0}^{n-1} y_{m}$ satisfying all the boundary conditions. It is clearly that $\psi_{n}(a)=y(a)$. For boundary condition $x=b$, the following scheme was proposed

$$
y_{0}(b)=y(b), \quad y_{n}(b)=0, \quad n \geq 1
$$

However, since each constant $c_{n}$ is determined by solving (1.12), this scheme also require additional computational work.

In order to avoid solving such nonlinear algebraic equations for a two-point boundary value problems, Jang [26] introduced extended ADM for nonsingular problems with Dirichlet boundary condition. Khuri and Sayfy [27] applied a novel approach based on the mixed decomposition spline for solving singular problems arising in physiology. The method proposed by Ebaid [4] is based on the modification of Lesnic's work [28]. Later, Duan and Rach [29] introduced special modified inverse linear integral operators for higher order boundary value problem.

To the best of our knowledge, no one has applied ADM to solve a class of doubly singular two-point boundary value problem (1.1) with Types 1 and 2 boundary conditions. 
In this work, a new approach based on ADM and new integral operator are proposed for solving doubly singular two-point boundary value problems (1.1) with Types 1 and 2. To set up the modified scheme, we first consider DSBVP (1.1) and use all the boundary conditions and Adomian decomposition method to establish the recursion scheme. Thus a modified recursion scheme is developed which does not require the computation of undermined coefficients, whereas most of previous recursive schemes do require the computation of undermined coefficients (see [23-25]). In fact, the proposed recursion scheme is useful for solving problem (1.1) whether they are linear or nonlinear singular (nonsingular). The main advantage of the method is that it provides a direct scheme for solving the singular boundary value problem, that is, without linearization and discretization. Numerical results are presented to demonstrate the effectiveness of proposed recursive scheme. The symbolic and numerical computations have been performed using "MATHEMATICA" software.

The organization of the paper is as follows. Section 2 presents the inverse integral operator with modified Adomian decomposition method for doubly singular boundary value problems of (1.1) with Types 1 and 2 boundary conditions. In Section 3, the convergence of the method is discussed. In Section 4, we illustrate our method with numerical results along with graphical representation.

\section{Modified Adomian Decomposition Method}

In this section, we establish two modified recursive schemes for solving doubly singular boundary value problems (1.1) with Types 1 and 2 boundary conditions.

We again write (1.1) with Type 1 boundary conditions as:

$$
\begin{gathered}
\left(p(t) y^{\prime}(t)\right)^{\prime}=q(t) f(t, y(t)), \quad 0<t \leq 1, \\
y(0)=a_{1}, \quad y(1)=c_{1},
\end{gathered}
$$

which can be rewritten as

$$
L y(t)=q(t) N y(t),
$$

where $L y(t)=\left(p(t) y^{\prime}(t)\right)^{\prime}$ is the linear differential operator to be inverted and $N y(t)=$ $f(t, y(t))$ is an analytic nonlinear operator. It is also assumed that the solution of the problem (2.1) exits and unique. A twofold integral operator $L^{-1}(\cdot)$, regarded as the inverse operator of $L(\cdot)$, is proposed as

$$
L^{-1}(\cdot)=\int_{0}^{t} \frac{1}{p(s)} \int_{s}^{1}(\cdot) d x d s
$$


To establish the recursive scheme, we operate $L^{-1}(\cdot)$ on the left hand side of (2.1) and use initial condition $y(0)=a_{1}$; we have

$$
\begin{aligned}
L^{-1}\left[\left(p(t) y^{\prime}(t)\right)^{\prime}\right] & =\int_{0}^{t} \frac{1}{p(s)} \int_{s}^{1}\left(p(x) y^{\prime}(x)\right)^{\prime} d x d s \\
& =c_{2} \int_{0}^{t} \frac{1}{p(s)} d s-y(t)+a_{1}, \\
L^{-1}\left[\left(p(t) y^{\prime}(t)\right)^{\prime}\right] & =c_{2} \int_{0}^{t} \frac{1}{p(s)} d s-y(t)+a_{1},
\end{aligned}
$$

where $c_{2}=p(1) y^{\prime}(1)$.

We again operate the inverse operator $L^{-1}(\cdot)$ on both sides of (2.1) and use (2.5), yielding

$$
y(t)=a_{1}+c_{2} \int_{0}^{t} \frac{1}{p(s)} d s-\int_{0}^{t} \frac{1}{p(s)} \int_{s}^{1} q(t) N y(t) d x d s
$$

For simplicity, we set $h(t)=\int_{0}^{t}(1 / p(s)) d s$ and $\left[L^{-1}(\cdot)\right]_{t=1}=\int_{0}^{1}(1 / p(s)) \int_{s}^{1}(\cdot) d x d s$.

Then (2.6) can be written as

$$
y(t)=a_{1}+c_{2} h(t)-\left[L^{-1} q(t) N y(t)\right]
$$

Using the boundary condition $y(1)=c_{1}$ in $(2.7)$, we get

$$
c_{2}=\frac{c_{1}-a_{1}}{h(1)}+\frac{1}{h(1)}\left[L^{-1} q(t) N y(t)\right]_{t=1} .
$$

By using $c_{2}$ into (2.7), we obtain

$$
y(t)=a_{1}+\frac{c_{1}-a_{1}}{h(1)} h(t)+\frac{h(t)}{h(1)}\left[L^{-1} q(t) N y(t)\right]_{t=1}-\left[L^{-1} q(t) N y(t)\right]
$$

It is important to note that the right hand side of (2.9) does not contain any unknown constants.

The equation (2.9) can be rewritten in the following operator equation form as

$$
y=f+M(y)
$$

where

$$
f=a_{1}+\frac{c_{1}-a_{1}}{h(1)} h(t), \quad M(y)=\frac{h(t)}{h(1)}\left[L^{-1} q(t) N y(t)\right]_{t=1}-\left[L^{-1} q(t) N y(t)\right]
$$


Next, the solution $y(t)$ and the nonlinear function $N y(t)$ are decomposed by infinite series of the form

$$
y(t)=\sum_{n=0}^{\infty} y_{n}(t), \quad N y(t)=\sum_{n=0}^{\infty} A_{n}\left(y_{0}(t), y_{1}(t), \ldots, y_{n}(t)\right)
$$

respectively, where $A_{n}\left(y_{0}(t), y_{1}(t), \ldots, y_{n}(t)\right)$ are Adomian polynomials [19].

Substituting the series (2.12) into (2.9) gives

$$
\sum_{n=0}^{\infty} y_{n}(t)=a_{1}+\frac{c_{1}-a_{1}}{h(1)} h(t)+\frac{h(t)}{h(1)}\left[L^{-1} q(t) \sum_{n=0}^{\infty} A_{n}\right]_{t=1}-\left[L^{-1} q(t) \sum_{n=0}^{\infty} A_{n}\right]
$$

Using the above equation (2.13), the solution components $y_{0}, y_{1}, \ldots, y_{n}$ are determined by the following recursive scheme:

$$
\begin{gathered}
y_{0}(t)=a_{1}+\frac{c_{1}-a_{1}}{h(1)} h(t), \\
y_{n+1}(t)=\frac{h(t)}{h(1)}\left[L^{-1} q(t) A_{n}\right]_{t=1}-\left[L^{-1} q(t) A_{n}\right], \quad n \geq 0, \\
\text { or } \\
y_{n+1}(t)=\frac{h(t)}{h(1)} \int_{0}^{1} \frac{1}{p(s)} \int_{s}^{1} q(t) A_{n} d x d s-\int_{0}^{t} \frac{1}{p(s)} \int_{s}^{1} q(t) A_{n} d x d s, \quad n \geq 0 .
\end{gathered}
$$

We further modify the above algorithm (2.14) to get a more efficient and economic algorithm. To obtain modified algorithm, the zeroth component $y_{0}$ is divided into the sum of two parts, namely, $f_{0}+f_{1}$, where $f_{0}=a_{1}, f_{1}=\left(\left(c_{1}-a_{1}\right) / h(1)\right) h(t)$. The first part, $f_{0}$, is kept in $y_{0}$ and the rest part, $f_{1}$, is added to $y_{1}$. Thus, the zeroth component will be $y_{0}=a_{1}$ in modified algorithm as

$$
\begin{aligned}
y_{0}(t) & =a_{1} \\
y_{1}(t) & =\frac{c_{1}-a_{1}}{h(1)} h(t)+\frac{h(t)}{h(1)}\left[L^{-1} q(t) A_{0}\right]_{t=1}-\left[L^{-1} q(t) A_{0}\right], \\
& \vdots \\
y_{n+1}(t) & =\frac{h(t)}{h(1)}\left[L^{-1} q(t) A_{n}\right]_{t=1}-\left[L^{-1} q(t) A_{n}\right], \quad n \geq 0 .
\end{aligned}
$$

Note that new modified recursion scheme (2.15) does not require the computation of undermined coefficients, since this algorithm does not contain any undermined coefficient. This slight change plays a major role for minimizing the size of calculations. 
Now, we again consider the same problem (1.1) with Type 2 boundary conditions:

$$
\begin{gathered}
\left(p(t) y^{\prime}(t)\right)^{\prime}=q(t) f(t, y(t)), \quad 0<t \leq 1, \\
y^{\prime}(0)=0, \quad a y(1)+b y^{\prime}(1)=c,
\end{gathered}
$$

which can be rewritten as

$$
L y(t)=q(t) N y(t)
$$

where $L y(t)=\left(p(t) y^{\prime}(t)\right)^{\prime}$ is the linear differential operator to be inverted and $N y(t)=$ $f(t, y(t))$ is an analytic nonlinear operator. It is also assumed that the solution of problem (2.16) exits and is unique. In this case, we propose the following inverse integral operator:

$$
L^{-1}(\cdot)=\int_{t}^{1} \frac{1}{p(s)} \int_{0}^{s}(\cdot) d x d s
$$

To setup an algorithm, we operate the inverse operator $L^{-1}(\cdot)$ on the left hand side of (2.16) and using the boundary condition $y^{\prime}(0)=0$, we have

$$
\begin{aligned}
L^{-1}\left[\left(p(t) y^{\prime}(t)\right)^{\prime}\right] & =\int_{t}^{1} \frac{1}{p(s)} \int_{0}^{s}\left(p(x) y^{\prime}(x)\right)^{\prime} d x d s \\
& =\int_{t}^{1} y^{\prime}(s) d s \\
L^{-1}\left[\left(p(t) y^{\prime}(t)\right)^{\prime}\right] & =c_{0}-y(t)
\end{aligned}
$$

where $c_{0}=y(1)$. have

By operating the inverse operator $L^{-1}(\cdot)$ on both sides of (2.16) and using (2.20) we

$$
y(t)=c_{0}-\int_{t}^{1} \frac{1}{p(s)} \int_{0}^{s} q(t) N y(t) d x d s
$$

Thus (2.21) can be written as

$$
y(t)=c_{0}-\left[L^{-1} q(t) N y(t)\right]
$$

Using boundary condition $a y(1)+b y^{\prime}(1)=c$ into (2.22), we obtain

$$
y(t)=\frac{c}{a}-\frac{b}{a p(1)} \int_{0}^{1} q(t) N y(t) d t-\left[L^{-1} q(t) N y(t)\right] .
$$


The equation (2.23) may be rewritten in operator equation form

$$
y=f+M(y)
$$

where

$$
f=\frac{c}{a}, \quad M(y)=-\frac{b}{a p(1)} \int_{0}^{1} q(t) N y(t) d x-\left[L^{-1} q(t) N y(t)\right]
$$

Substituting the series (2.12) into (2.23) gives

$$
\sum_{n=0}^{\infty} y_{n}(t)=\frac{c}{a}-\frac{b}{a p(1)} \int_{0}^{1} q(t) \sum_{n=0}^{\infty} A_{n} d t-\left[L^{-1} q(t) \sum_{n=0}^{\infty} A_{n}\right] .
$$

Upon matching both sides of (2.26), the solution components $y_{0}, y_{1}, \ldots, y_{n}$ can be determined by the following modified recursive scheme:

$$
\begin{gathered}
y_{0}(t)=\frac{c}{a} \\
y_{n+1}(t)=-\frac{b}{a p(1)} \int_{0}^{1} q(t) A_{n} d t-\left[L^{-1} q(t) A_{n}\right], \quad n \geq 0 .
\end{gathered}
$$

Thus, we have established two modified recursive schemes which give the complete determination of solution components $y_{n}$, and hence the approximate series solution $\psi_{n}(t)$ can be obtained for the singular boundary value problems (1.1) with Types 1 and 2 boundary conditions. For numerical purpose, the truncated $n$-term approximate series solution is given by

$$
\psi_{n}(t)=\sum_{m=0}^{n-1} y_{m}(t)
$$

Note that the truncated $n$-term approximate series solution is obtained by adding $n$ solutions components $y_{0}, y_{1}, \ldots, y_{n-1}$, that is, $\psi_{n}=\sum_{m=0}^{n-1} y_{m}$.

\section{Convergence of Method}

In this section, we discuss the convergence analysis of modified ADM for doubly singular boundary value problem (1.1).

Many authors [30-33] established the convergence of ADM for differential and integral equations. The first proof of convergence of ADM for the general functional equation was given by Cherruault [31]. Cherruault and Adomian [32] proposed a new convergence proof of Adomian decomposition method for the general nonlinear functional equation based on the properties of convergent series. Recently, Hosseini and Nasabzadeh [33] introduced a simple technique to determine the rate of convergence of ADM for initial value problem. 
Now, we discuss the convergence of doubly singular two-point boundary value problems (1.1) with Types 1 and 2 boundary conditions. To do so, note that (2.9) and (2.23) may be written in the operator equation form

$$
y=f+M(y),
$$

where $M$ is an operator from a Banach space $C[0,1]$ to $C[0,1], f$ is a given function in $C[0,1]$, and we are looking for $y \in C[0,1]$ satisfying (3.1).

For Type 1 boundary conditions, we have

$$
\begin{gathered}
f=a_{1}+\frac{\left(c_{1}-a_{1}\right)}{h(1)} h(t), \\
M(y)=\frac{h(t)}{h(1)}\left[L^{-1} q(t) N y(t)\right]_{t=1}-\left[L^{-1} q(t) N y(t)\right] .
\end{gathered}
$$

For Type 2 boundary conditions, $f$ and $M$ are given as

$$
\begin{gathered}
f=\frac{c}{a} \\
M(y)=-\frac{b}{a p(1)} \int_{0}^{1} q(t) N y(t) d t-\left[L^{-1} q(t) N y(t)\right] .
\end{gathered}
$$

Let the solution $y$ and the nonlinear function $N y$ be considered as sum of series

$$
y=\sum_{n=0}^{\infty} y_{n}, \quad N y=\sum_{n=0}^{\infty} A_{n}\left(y_{0}, y_{1}, \ldots, y_{n}\right)
$$

where $A_{n}\left(y_{0}, y_{1}, \ldots, y_{n}\right)$ are Adomian polynomials [19].

Substituting the series (3.6) into the operator equation (3.1) yields

$$
\begin{gathered}
\sum_{n=0}^{\infty} y_{n}=f+\sum_{n=0}^{\infty} B_{n}\left(y_{0}, \ldots, y_{n}\right), \\
y_{0}+y_{1}+\cdots+y_{n}+\cdots=f+B_{0}\left(y_{0}\right)+\cdots+B_{n-1}\left(y_{0}, \ldots, y_{n-1}\right)+\cdots,
\end{gathered}
$$

where

$$
\begin{aligned}
& \sum_{n=0}^{\infty} B_{n}\left(y_{0}, \ldots, y_{n}\right)=\frac{h(t)}{h(1)}\left[L^{-1} q(t) \sum_{n=0}^{\infty} A_{n}\right]_{t=1}-\left[L^{-1} q(t) \sum_{n=0}^{\infty} A_{n}\right], \\
& \sum_{n=0}^{\infty} B_{n}\left(y_{0}, \ldots, y_{n}\right)=-\frac{b}{a p(1)} \int_{0}^{1} q(t) \sum_{n=0}^{\infty} A_{n} d t-\left[L^{-1} q(t) \sum_{n=0}^{\infty} A_{n}\right],
\end{aligned}
$$

for Types 1 and 2 boundary conditions, respectively. 
Upon matching both sides of (3.7), we obtain the following scheme:

$$
\begin{aligned}
y_{0}= & f, \\
y_{1} & =B_{0}\left(y_{0}\right), \\
& \vdots \\
y_{n+1} & =B_{n}\left(y_{0}, \ldots, y_{n}\right),
\end{aligned}
$$

Let $\psi_{n}=\sum_{m=0}^{n-1} y_{m}$ be approximate solution, then the modified ADM for (3.1) is equivalent to the following problem:

$$
\begin{gathered}
y_{0}=f, \\
\psi_{n+1}=y_{0}+M\left(\psi_{n}\right), \quad n \geq 0 .
\end{gathered}
$$

Finding the solution of (3.1) is equivalent to finding the sequence $\psi_{n}$ such that $\psi_{n}=y_{0}+y_{1}+$ $\cdots+y_{n-1}$ satisfies (3.10).

Theorem 3.1. Let $M(y)$ be the nonlinear operator defined by (3.3) or (3.5) which satisfies the Lipschitz condition $\|M(\varphi)-M(\xi)\| \leq \delta\|\varphi-\xi\|$, for all $\varphi, \xi \in C[0,1]$ with Lipschitz constant $\delta$, $0 \leq \delta<1$. If $\left\|y_{0}\right\|<\infty$, then there holds $\left\|y_{k+1}\right\| \leq \delta\left\|y_{k}\right\|$, for all $k \in \mathbb{N} \cup\{0\}$ and the sequence $\left\{\psi_{n}\right\}$ defined by (3.10) converges to $y=\sum_{n=0}^{\infty} y_{n}=\lim _{n \rightarrow \infty} \psi_{n}=\psi$.

Proof. Since

$$
\psi_{1}=y_{0}+y_{1}, \quad \psi_{2}=y_{0}+y_{1}+y_{2}, \ldots, \quad \psi_{n}=y_{0}+y_{1}+y_{2}+\cdots+y_{n}, \ldots,
$$

we have $y_{k+1}=\psi_{k+1}-\psi_{k}, k=1,2, \ldots$

We now show that the sequence $\left\{\psi_{n}\right\}$ is convergent. To prove this, it is sufficient to show that $\left\{\psi_{n}\right\}$ is cauchy sequence in Banach space $C[0,1]$. Now using the nonlinearity of $M(y)$, we have

$$
\left\|y_{n+1}\right\|=\left\|\psi_{n+1}-\psi_{n}\right\|=\left\|M\left(\psi_{n}\right)-M\left(\psi_{n-1}\right)\right\| \leq \delta\left\|\psi_{n}-\psi_{n-1}\right\|=\delta\left\|y_{n}\right\|
$$

Thus we obtain

$$
\left\|y_{n+1}\right\| \leq \delta\left\|y_{n}\right\| \leq \delta^{2}\left\|y_{n-1}\right\| \cdots \leq \delta^{n+1}\left\|y_{0}\right\|
$$


Now for every $n, m \in \mathbb{N}$, and $n \geq m$, we have

$$
\begin{aligned}
\left\|\psi_{n}-\psi_{m}\right\| & =\left\|\left(\psi_{n}-\psi_{n-1}\right)+\left(\psi_{n-1}-\psi_{n-2}\right)+\cdots+\left(\psi_{m+1}-\psi_{m}\right)\right\| \\
& \leq\left\|\psi_{n}-\psi_{n-1}\right\|+\left\|\psi_{n-1}-\psi_{n-2}\right\|+\cdots+\left\|\psi_{m+1}-\psi_{m}\right\| \\
& \leq \delta^{n}\left\|y_{0}\right\|+\delta^{n-1}\left\|y_{0}\right\|+\cdots+\delta^{m+1}\left\|y_{0}\right\| \\
& \leq \delta^{m+1}\left(1+\delta+\delta^{2}+\cdots+\delta^{n-m-1}\right)\left\|y_{0}\right\| .
\end{aligned}
$$

As we know $\sum_{j=0}^{\infty} \delta^{j}$ is geometric series with common ration $\delta, 0 \leq \delta<1$. Therefore, we have

$$
\left\|\psi_{n}-\psi_{m}\right\| \leq \frac{\delta^{m+1}}{1-\delta}\left\|y_{0}\right\|
$$

which converges to zero, that is, $\left\|\psi_{n}-\psi_{m}\right\| \rightarrow 0$, as $m \rightarrow \infty$. This implies that there exits $\psi$ such that $\lim _{n \rightarrow \infty} \psi_{n}=\psi$. But, we have $y=\sum_{n=0}^{\infty} y_{n}=\lim _{n \rightarrow \infty} \psi_{n}$, that is, $y=\psi$ which is solution of (3.1). Hence, we have convergence in norm.

\section{Numerical Examples and Discussion}

In this section, we consider four examples to demonstrate the effectiveness of proposed recursive schemes (2.15) and (2.27). The numerical results are compared with known results and maximum absolute error is also calculated. We also plot approximate and exact solutions to show how approximate solutions converge to exact solution.

Example 4.1. Consider linear two-point boundary value problem [6]

$$
\begin{gathered}
\left(t^{\alpha} y^{\prime}\right)^{\prime}=\beta t^{\alpha+\beta-2}\left(\beta t^{\beta}+\alpha+\beta-1\right) y, \quad 0<t \leq 1, \\
y(0)=1, \quad y(1)=e,
\end{gathered}
$$

with exact solution $y(t)=e^{t^{\beta}}$. For every $\alpha>0$ and $\beta>0,(4.1)$ is called singular. 
In particular, if $\alpha=0.5, \beta=1$, that is, $p(t)=t^{0.5}$ and $q(t)=t^{-0.5}$ then problem (4.1) is called doubly singular boundary value problem. Applying modified recursive scheme (2.15) to (4.1), the solution components are obtained as

$$
\begin{aligned}
y_{0}= & 1 \\
y_{1}= & 0.384948 t^{0.5}+t+0.333333 t^{2} \\
y_{2}= & -0.551338 t^{0.5}+0.128316 t^{1.5}+0.166667 t^{2}+0.076989 t^{2.5}+0.155556 t^{3}+0.023809 t^{4}, \\
y_{3}= & 0.224316 t^{0.5}-0.183779 t^{1.5}-0.097435 t^{2.5}+0.011111 t^{3}+0.015886 t^{3.5}+0.017460 t^{4} \\
& +0.004277 t^{4.5}+0.007442 t^{5}+0.000721 t^{6}, \\
y_{4}= & -0.077011 t^{0.5}+0.074771 t^{1.5}+0.026485 t^{2.5}-0.022142 t^{3.5}+0.000396 t^{4} \\
& -0.004971 t^{4.5}+0.000881 t^{5}+0.000655 t^{5.5}+0.0006418 t^{6}+0.0001096 t^{6.5} \\
& +0.0001715 t^{7}+0.000012 t^{8} \\
\vdots &
\end{aligned}
$$

For numerical purpose, the approximate series solution is

$$
\begin{aligned}
\psi_{6}= & -0.002003 t^{0.5}+t+0.002065 t^{1.5}+0.5 t^{2}+0.000603 t^{2.5}+0.166667 t^{3} \\
& -0.0006957 t^{3.5}+0.041666 t^{4}-0.0000457 t^{4.5}+0.0083333 t^{5}+0.0000802 t^{5.5} \\
& +0.001388 t^{6}+1.0684407 \times 10^{-6} t^{6.5}+0.000198 t^{7}-4.663181 \times 10^{-6} t^{7.5} \\
& +0.000024 t^{8}-3.885381 \times 10^{-8} t^{8.5}+2.745287 \times 10^{-6} t^{9}+1.676726 \times 10^{-7} t^{9.5} \\
& +2.664448 \times 10^{-7} t^{10}+1.5360215 \times 10^{-8} t^{10.5}+2.063867 \times 10^{-8} t^{11} \\
& +9.172396 \times 10^{-10} t^{12} .
\end{aligned}
$$

Now, we define error function as $E_{n}(t)=\psi_{n}(t)-y(t)$ and the maximum absolute errors as

$$
\left\|E_{n}\right\|_{\infty}=\max _{0<t \leq 1}\left|E_{n}(t)\right|
$$

For different values of $\alpha$ and $\beta$, the maximum absolute error $\left\|E_{n}\right\|_{\infty}$, for $n=4,6$, and 8 , is shown in Tables 1 and 2, and we observe that when $n$ increases the error decreases. On the other hand, Chawla and Katti [6] applied $M_{3}$ method based on three evaluations of $f$ to solve the same problem in which a huge amount of computation work is needed to obtain the numerical solution. The results are compared with our approximate series solution in Table 3. Furthermore, the approximate series solutions $\psi_{2}, \psi_{4}$ and exact solution $y$ are plotted in Figures 1 and 2. It can be seen from the figures that series solution is very close to exact solution. 
Table 1: Maximum error of Example 4.1, when $\beta=1$.

\begin{tabular}{lccc}
\hline$\alpha$ & $\left\|E_{4}\right\|_{\infty}$ & $\left\|E_{6}\right\|_{\infty}$ & $\left\|E_{8}\right\|_{\infty}$ \\
\hline 0.25 & $2.3211 \times 10^{-3}$ & $4.2210 \times 10^{-5}$ & $2.4843 \times 10^{-6}$ \\
0.5 & $2.3151 \times 10^{-3}$ & $6.1226 \times 10^{-5}$ & $3.2320 \times 10^{-6}$ \\
0.75 & $3.5315 \times 10^{-3}$ & $7.0126 \times 10^{-5}$ & $2.2117 \times 10^{-6}$ \\
\hline
\end{tabular}

Table 2: Maximum error of Example 4.1, when $\beta=5$.

\begin{tabular}{lccc}
\hline$\alpha$ & $\left\|E_{4}\right\|_{\infty}$ & $\left\|E_{6}\right\|_{\infty}$ & $\left\|E_{8}\right\|_{\infty}$ \\
\hline 0.25 & $2.2419 \times 10^{-3}$ & $3.3304 \times 10^{-5}$ & $3.5127 \times 10^{-6}$ \\
0.5 & $1.3307 \times 10^{-3}$ & $5.0264 \times 10^{-5}$ & $4.6572 \times 10^{-6}$ \\
0.75 & $4.2104 \times 10^{-3}$ & $4.3231 \times 10^{-5}$ & $2.1260 \times 10^{-6}$ \\
\hline
\end{tabular}

Example 4.2. Consider nonlinear singular two-point boundary value problem

$$
\begin{gathered}
\left(t^{\alpha} y^{\prime}\right)^{\prime}=t^{\alpha+\beta-2} e^{y}\left(t^{\beta} e^{y}-\alpha-\beta+1\right), \quad 0<t \leq 1, \\
y(0)=\ln \left(\frac{\beta}{4}\right), \quad y(1)=\ln \left(\frac{\beta}{5}\right),
\end{gathered}
$$

with exact solution $y(t)=\ln \left(\beta /\left(4+t^{\beta}\right)\right)$ and for any real $\alpha>0$ and $\beta>0$, this equation is called singular.

If $\alpha=0.25, \beta=1$, that is, $p(t)=t^{0.25}, q(t)=t^{-0.75}$, then problem (4.5) is called doubly singular boundary value problem.

Now we apply recursive scheme (2.15) to obtain the solution components as

$$
\begin{aligned}
y_{0}= & -1.38629, \\
y_{1}= & 0.001856 t^{0.75}-0.25 t+0.025 t^{2}, \\
y_{2}= & -0.001605 t^{0.75}-0.000066 t^{1.75}+0.00625 t^{2}+0.000042 t^{2.75}-0.004861 t^{3}+0.000240 t^{4}, \\
y_{3}= & -0.000283 t^{0.75}+0.000057 t^{1.75}-2.461715 \times 10^{-8} t^{2.5}-0.000030 t^{2.75}-0.000347 t^{3} \\
& +4.475846 \times 10^{-8} t^{3.5}-0.000011 t^{3.75}+0.000714 t^{4}+8.882529 \times 10^{-7} t^{4.75} \\
& -0.000103 t^{5}+3.434065 \times 10^{-6} t^{6},
\end{aligned}
$$


Table 3: Maximum error of Example 4.1.

\begin{tabular}{lccc}
\hline \multicolumn{2}{c}{ Proposed method } & \multicolumn{2}{c}{ Chawla and Katti [6] } \\
$\alpha, \beta$ & $\left\|E_{8}\right\|_{\infty}$ & $N$ & error \\
\hline$\alpha=0.50, \beta=4.0$ & $2.4003 \times 10^{-6}$ & 128 & $1.80 \times 10^{-4}$ \\
$\alpha=0.75, \beta=3.75$ & $5.1431 \times 10^{-6}$ & 128 & $1.80 \times 10^{-4}$ \\
\hline
\end{tabular}

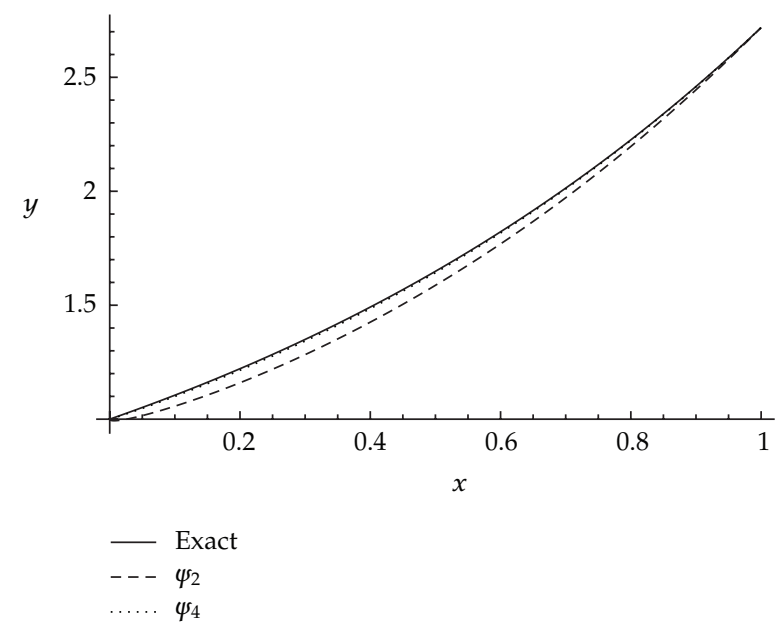

Figure 1: Comparison of exact $y$ and approximate $\psi_{2}, \psi_{4}$ solutions of Example 4.1, $\alpha=0.50, \beta=1$.

To compare the results numerically, the 6-term truncated series solution is given by

$$
\begin{aligned}
\psi_{6}= & -1.38629-3.372935 \times 10^{-7} x^{0.75}-0.25 t-1.108619 \times 10^{-7} t^{1.75}+0.03125 t^{2} \\
& +7.059037 \times 10^{-9} t^{2.5}-3.299920 \times 10^{-8} t^{2.75}-0.005208 t^{3}+1.307442 \times 10^{-11} t^{3.25} \\
& -1.557418 \times 10^{-8} t^{3.5}+1.289457 \times 10^{-7} t^{3.75}+0.000976 t^{4}-5.530529 \times 10^{-11} t^{4.25} \\
& +1.078097 \times 10^{-8} t^{4.5}+3.090869 \times 10^{-7} t^{4.75}-0.000195 t^{5}-1.480660 \times 10^{-11} t^{5.25} \\
& +1.980188 \times 10^{-9} t^{5.5}-3.668865 \times 10^{-7} t^{5.75}+0.0000405 t^{6}+2.567699 \times 10^{-12} t^{6.25} \\
& -1.009792 \times 10^{-9} t^{6.5}+1.315036 \times 10^{-7} t^{6.75}-7.607217 \times 10^{-6} t^{7} \\
& +5.654798 \times 10^{-11} t^{7.5}-1.378289 \times 10^{-8} t^{7.75}+8.830360 \times 10^{-7} t^{8}+4.154631 \\
& \times 10^{-10} t^{8.75}-4.867214 \times 10^{-8} t^{9}+9.674748 \times 10^{-10} t^{10} .
\end{aligned}
$$




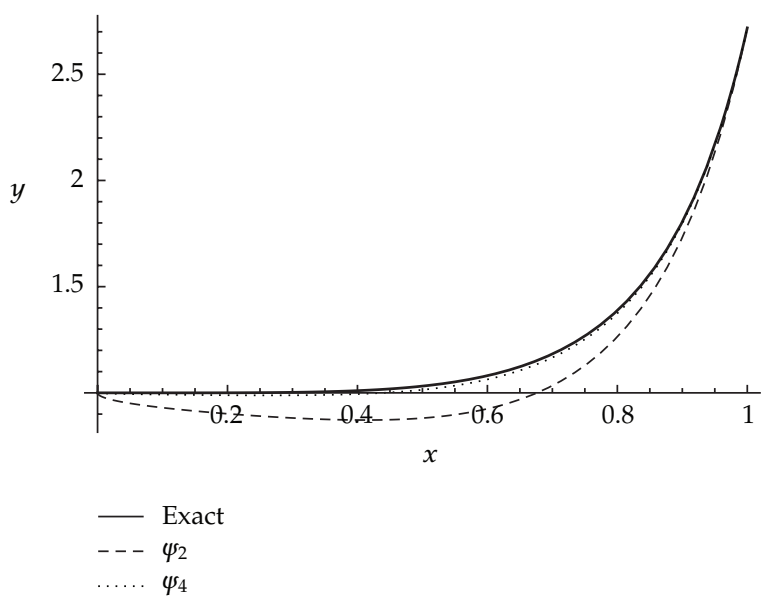

Figure 2: Comparison of exact $y$ and approximate $\psi_{2}, \psi_{4}$ solutions of Example $4.1, \alpha=0.50, \beta=5$.

Table 4: Maximum error for Example 4.2, when $\beta=1$.

\begin{tabular}{lccc}
\hline$\alpha$ & $\left\|E_{4}\right\|_{\infty}$ & $\left\|E_{6}\right\|_{\infty}$ & $\left\|E_{8}\right\|_{\infty}$ \\
\hline 0.25 & $1.3889 \times 10^{-4}$ & $2.0435 \times 10^{-6}$ & $2.5693 \times 10^{-8}$ \\
0.5 & $5.2987 \times 10^{-4}$ & $1.1481 \times 10^{-6}$ & $1.1616 \times 10^{-8}$ \\
0.75 & $2.4545 \times 10^{-4}$ & $3.7571 \times 10^{-7}$ & $2.1654 \times 10^{-9}$ \\
\hline
\end{tabular}

Similarly, for $\alpha=0.5, \beta=1$, that is, $p(t)=t^{0.5}$ and $q(t)=t^{-0.5}$, the solution components are obtained using the scheme (2.15) as

$$
\begin{aligned}
y_{0}= & -1.38629, \\
y_{1}= & 0.006023 t^{0.5}-0.25 t+0.020833 t^{2}, \\
y_{2}= & -0.005737 t^{0.5}-0.000501 t^{1.5}+0.010416 t^{2}+0.000150 t^{2.5}-0.004513 t^{3}+0.000186 t^{4}, \\
y_{3}= & -0.000302 t^{0.5}+0.000478 t^{1.5}-7.557899 \times 10^{-7} t^{2}-0.000093 t^{2.5}-0.000693 t^{3} \\
& -0.000045 t^{3.5}+0.0007378 t^{4}+2.788479 \times 10^{-6} t^{4.5}-0.0000851 t^{5}+2.348635 \times 10^{-6} t^{6},
\end{aligned}
$$

The maximum absolute error $\left\|E_{n}\right\|_{\infty}$, for $n=4,6$, and 8 , is listed in Tables 4 and 5 . As the value of $n$ increases, the maximum absolute error decreases. In addition, to verify how close is our approximate series solutions $\psi_{2}, \psi_{4}$ to exact solution $y$, we have plotted approximate solution and exact solution in Figures 3 and 4. It is shown from figures that the approximate series solution $\psi_{4}$ is very near to the exact solution $y$. 
Table 5: Maximum error for Example 4.2, when $\beta=5$.

\begin{tabular}{lccc}
\hline$\alpha$ & $\left\|E_{4}\right\|_{\infty}$ & $\left\|E_{6}\right\|_{\infty}$ & $\left\|E_{8}\right\|_{\infty}$ \\
\hline 0.25 & $6.5109 \times 10^{-4}$ & $2.6137 \times 10^{-7}$ & $6.0161 \times 10^{-8}$ \\
0.5 & $8.8947 \times 10^{-4}$ & $1.1610 \times 10^{-6}$ & $7.1746 \times 10^{-8}$ \\
0.75 & $7.6596 \times 10^{-4}$ & $5.2644 \times 10^{-7}$ & $6.3210 \times 10^{-8}$ \\
\hline
\end{tabular}

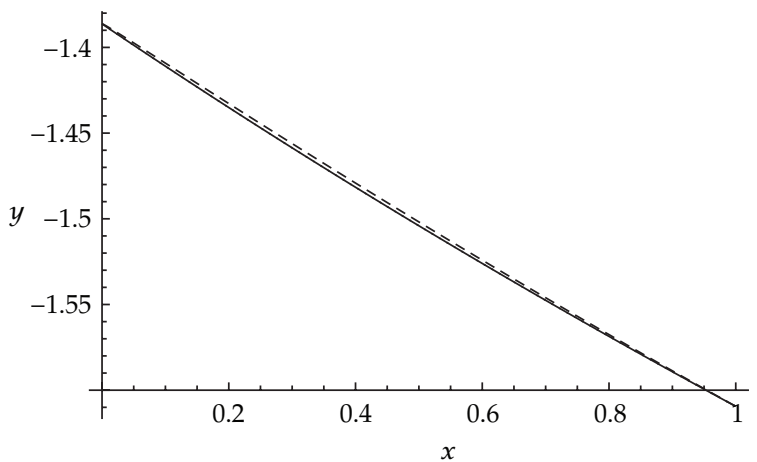

$\begin{array}{ll} & \text { Exact } \\ --- & \psi_{2} \\ \ldots \ldots & \psi_{4}\end{array}$

Figure 3: Comparison of exact $y$ and approximate $\psi_{2}, \psi_{4}$ solutions of Example 4.2 , when $\alpha=0.50, \beta=1$.

Example 4.3. The heat conduction model in human head problem Duggan and Goodman [13] is

$$
\begin{gathered}
\left(t^{2} y^{\prime}\right)^{\prime}=-t^{2} e^{-y}, \quad 0<t \leq 1, \\
y^{\prime}(0)=0, \quad 2 y(1)+y^{\prime}(1)=0 .
\end{gathered}
$$

We apply the recursive scheme (2.27) to (4.9), where $p(t)=q(t)=t^{2}, f=e^{-y}, a=2, b=1$, and $c=0$ then scheme (2.27) for (4.9) becomes as

$$
\begin{gathered}
y_{0}=0, \\
y_{n+1}=\frac{1}{2} \int_{0}^{1} t^{2} A_{n} d t+\int_{t}^{1} s^{-2}\left(\int_{0}^{s} x^{2} A_{n} d x\right) d s, \quad n \geq 0 .
\end{gathered}
$$




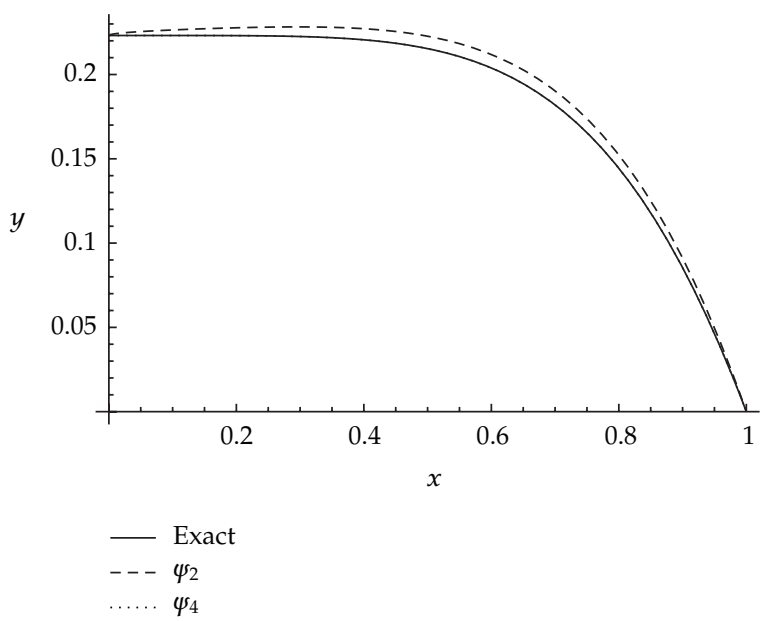

Figure 4: Comparison of exact $y$ and approximate $\psi_{2}, \psi_{4}$ solutions of Example 4.2, when $\alpha=0.50, \beta=5$.

We can easily determine the solution components from above scheme as

$$
\begin{aligned}
y_{0} & =0, \\
y_{1} & =0.333333-0.166667 t^{2}, \\
y_{2} & =-0.086111+0.055555 t^{2}-0.008333 t^{4}, \\
y_{3} & =0.032672-0.023611 t^{2}+0.005555 t^{4}-0.000529 t^{6}, \\
y_{4} & =-0.014584+0.011258 t^{2}-0.003287 t^{4}+0.000529 t^{6}-0.0000373 t^{8}, \\
& \vdots
\end{aligned}
$$

The truncated series solution $\psi_{8}$ is obtained by adding $y_{0}, y_{1}, \ldots, y_{6}$, given by

$$
\begin{aligned}
\psi_{8}= & 0.270259-0.127429 t^{2}-0.0047677 t^{4 .}-0.0002650 t^{6}-4.7439600 \times 10^{-6} t^{8} \\
& -2.4024518 \times 10^{-6} t^{10}+2.2847637 \times 10^{-7} t^{12}-3.485344 \times 10^{-8} t^{14} \\
& +2.384089 \times 10^{-9} t^{16}-1.1874334 \times 10^{-10} t^{18}
\end{aligned}
$$

The comparison of numerical results of solutions with known solution is given in Table 6. It can be noted that the approximate solution $\psi_{8}$ is very close to known solution [13]. However, Duggan and Goodman [13] utilized the maximum principle based on lower and an upper solutions technique in which a lot of calculation is required as we need to solve sequence of linear differential equation analytically. 
Table 6: Comparison of numerical results with known results of Example 4.3.

\begin{tabular}{ccccc}
\hline$t$ & $\psi_{6}$ & $\psi_{8}$ & $\psi_{10}$ & Solution in [13] \\
\hline 0 & 0.27073627 & 0.27025895 & 0.27010891 & 0.27035006 \\
0.2 & 0.26561546 & 0.26515412 & 0.26500927 & 0.26525434 \\
0.4 & 0.25016332 & 0.24974709 & 0.24961677 & 0.24986712 \\
0.6 & 0.22410365 & 0.22375398 & 0.22364491 & 0.22388597 \\
0.8 & 0.18695255 & 0.18668071 & 0.18659620 & 0.18679895 \\
1 & 0.13798246 & 0.13778979 & 0.13772998 & 0.13787263 \\
\hline
\end{tabular}

Example 4.4. The nonlinear singular boundary problem considered by Chawla et al. [34] is

$$
\begin{gathered}
y^{\prime \prime}+\frac{1}{t} y^{\prime}=-v e^{y}, \quad 0<t<1, \\
y^{\prime}(0)=0, \quad y(1)=0,
\end{gathered}
$$

with exact solution $y(t)=2 \ln \left((B+1) /\left(B t^{2}+1\right)\right)$, where $B=\left(4-v-2(4-2 v)^{1 / 2}\right) / v, v=1$.

In the above problem, we have $p(t)=q(t)=t$ and $f=-e^{y}, a=1, b=0, c=0$, by applying recursive scheme (2.27), we have following recursive scheme:

$$
\begin{gathered}
y_{0}=0 \\
y_{n+1}=\frac{0}{1} \int_{0}^{1} t A_{n} d t+\int_{t}^{1} s^{-1}\left(\int_{0}^{s} x A_{n} d x\right) d s, \quad n \geq 0 .
\end{gathered}
$$

We have successive solution components $y_{n}$ as

$$
\begin{aligned}
y_{0} & =0, \\
y_{1} & =0.25-0.25 t^{2}, \\
y_{2} & =0.046875-0.0625 t^{2}+0.015625 t^{4}, \\
y_{3} & =0.0130208-0.0195313 t^{2}+0.0078125 t^{4}-0.00130208 t^{6}, \\
& \vdots
\end{aligned}
$$

and then the truncated series is obtained as

$$
\begin{aligned}
\psi_{6}= & 0.316294-0.342438 t^{2}+0.0289497 t^{4}-0.003107 t^{6}+0.0003280 x^{8} \\
& -0.0000274 t^{10}+1.2715657 \times 10^{-6} t^{12}
\end{aligned}
$$

In order to check whether the approximate solution converges to exact solution $y$, we plot approximate solution $\psi_{n}$, for $n=2,4$ and exact solution $y$ in Figure 5. It is clear from the figure that the approximate solution $\psi_{4}$ converges rapidly to exact solution. The maximum 
Table 7: Numerical results and maximum error of Example 4.4.

\begin{tabular}{lccc}
\hline$n$ & $\left\|E_{n}\right\|_{\infty}$ & $n$ & Error in [34] \\
\hline 5 & $6.2129 \times 10^{-4}$ & 16 & $2.52 \times 10^{-3}$ \\
8 & $1.0919 \times 10^{-5}$ & 32 & $1.83 \times 10^{-4}$ \\
10 & $3.0638 \times 10^{-6}$ & 64 & $1.28 \times 10^{-5}$ \\
\hline
\end{tabular}

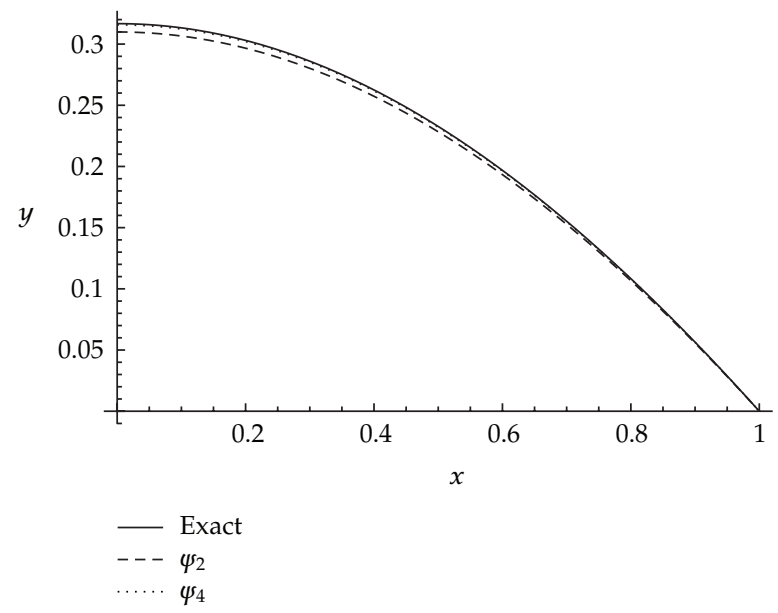

Figure 5: Comparison of exact $y$ and approximate $\psi_{2}, \psi_{4}$ solutions of Example 4.4.

absolute error $\left\|E_{n}\right\|_{\infty}$, for $n=5,8$, and 10 , is presented in Table 7 . It can be noted that Chawla et al. [34] employed fourth-order finite difference method to obtain the numerical solution in which a lot of computation work is required. However, the maximum absolute error obtained was $1.28 \times 10^{-5}$ using $N=64$.

\section{Conclusion}

In this paper, we we have illustrated how the modified recursive schemes (2.15) and (2.27) can be used to solve a class of doubly singular two-point boundary value problems (1.1) with Types 1 and 2 boundary conditions. The accuracy of the numerical results indicates that the method is well suited for the solution of this type problem. The main advantage of this approach is that it provides a direct scheme to obtain approximate solutions, and we have also shown graphically that these approximate solutions are almost identical to the analytic solution. Another advantage of a modified recursion scheme is that this does not require the computation of undermined coefficients, whereas most of previous recursive schemes do require the computation of undermined coefficients (see [23-25]). The method provides a reliable technique which requires less work compared to the traditional techniques such as finite difference method, Cubic spline method, and standard ADM. The numerical results of the examples are presented and only a few terms are required to obtain accurate solutions. By comparing the results with other existing methods, it has been proved that proposed modified ADM is a more powerful method for solving the singular problems (1.1). 


\section{References}

[1] L. E. Bobisud, "Existence of solutions for nonlinear singular boundary value problems," Applicable Analysis, vol. 35, no. 1-4, pp. 43-57, 1990.

[2] T. Aziz and M. Kumar, "A fourth-order finite-difference method based on non-uniform mesh for a class of singular two-point boundary value problems," Journal of Computational and Applied Mathematics, vol. 136, no. 1-2, pp. 337-342, 2001.

[3] M. M. Chawla and C. P. Katti, "A uniform mesh finite difference method for a class of singular twopoint boundary value problems," SIAM Journal on Numerical Analysis, vol. 22, no. 3, pp. 561-565, 1985.

[4] A. Ebaid, "A new analytical and numerical treatment for singular two-point boundary value problems via the Adomian decomposition method," Journal of Computational and Applied Mathematics, vol. 235, no. 8, pp. 1914-1924, 2011.

[5] M. Kumar, "A new finite difference method for a class of singular two-point boundary value problems," Applied Mathematics and Computation, vol. 143, no. 2-3, pp. 551-557, 2003.

[6] M. M. Chawla and C. P. Katti, "Finite difference methods and their convergence for a class of singular two-point boundary value problems," Numerische Mathematik, vol. 39, no. 3, pp. 341-350, 1982.

[7] M. M. Chawla and C. P. Katti, "A finite-difference method for a class of singular two-point boundary value problems," IMA Journal of Numerical Analysis, vol. 4, no. 4, pp. 457-466, 1984.

[8] M. M. Chawla, S. McKee, and G. Shaw, "Order 2 method for a singular two-point boundary value problem," BIT Numerical Mathematics, vol. 26, no. 3, pp. 318-326, 1986.

[9] S. R. K. Iyengar and P. Jain, "Spline finite difference methods for singular two point boundary value problems," Numerische Mathematik, vol. 50, no. 3, pp. 363-376, 1987.

[10] L. Thomas, "The calculation of atomic fields," in Mathematical Proceedings of the Cambridge Philosophical Society, vol. 23, pp. 542-548, Cambridge University Press, 1927.

[11] E. Fermi, "Un metodo statistico per la determinazione di alcune priorieta dell'atome," Rendicondi Accademia Nazionale de Lincei, vol. 32, no. 6, pp. 602-607, 1927.

[12] C. Y. Chan and Y. C. Hon, "A constructive solution for a generalized Thomas-Fermi theory of ionized atoms," Quarterly of Applied Mathematics, vol. 45, no. 3, pp. 591-599, 1987.

[13] R. Duggan and A. Goodman, "Pointwise bounds for a nonlinear heat conduction model of the human head," Bulletin of Mathematical Biology, vol. 48, no. 2, pp. 229-236, 1986.

[14] G. W. Reddien, "Projection methods and singular two point boundary value problems," Numerische Mathematik, vol. 21, pp. 193-205, 1974.

[15] M. M. Chawla and P. N. Shivakumar, "On the existence of solutions of a class of singular nonlinear two-point boundary value problems," Journal of Computational and Applied Mathematics, vol. 19, no. 3, pp. 379-388, 1987.

[16] D. R. Dunninger and J. C. Kurtz, "Existence of solutions for some nonlinear singular boundary value problems," Journal of Mathematical Analysis and Applications, vol. 115, no. 2, pp. 396-405, 1986.

[17] R. K. Pandey and A. K. Verma, "A note on existence-uniqueness results for a class of doubly singular boundary value problems," Nonlinear Analysis: Theory, Methods E Applications, vol. 71, no. 7-8, pp. 3477-3487, 2009.

[18] G. Adomian, Solving Frontier Problems of Physics: The Decomposition Method, vol. 60 of Fundamental Theories of Physics, Kluwer Academic, Dordrecht, The Netherlands, 1994.

[19] G. Adomian and R. Rach, "Inversion of nonlinear stochastic operators," Journal of Mathematical Analysis and Applications, vol. 91, no. 1, pp. 39-46, 1983.

[20] G. Adomian and R. Rach, "A new algorithm for matching boundary conditions in decomposition solutions," Applied Mathematics and Computation, vol. 58, no. 1, pp. 61-68, 1993.

[21] G. Adomian and R. Rach, "Modified decomposition solution of linear and nonlinear boundary-value problems," Nonlinear Analysis: Theory, Methods E Applications, vol. 23, no. 5, pp. 615-619, 1994.

[22] A.-M. Wazwaz, "Approximate solutions to boundary value problems of higher order by the modified decomposition method," Computers \& Mathematics with Applications, vol. 40, no. 6-7, pp. 679-691, 2000.

[23] A. M. Wazwaz, "A reliable algorithm for obtaining positive solutions for nonlinear boundary value problems," Computers \& Mathematics with Applications, vol. 41, no. 10-11, pp. 1237-1244, 2001.

[24] M. Benabidallah and Y. Cherruault, "Application of the Adomian method for solving a class of boundary problems," Kybernetes, vol. 33, no. 1, pp. 118-132, 2004.

[25] M. Inc and D. J. Evans, "The decomposition method for solving of a class of singular two-point boundary value problems," International Journal of Computer Mathematics, vol. 80, no. 7, pp. 869-882, 2003. 
[26] B. Jang, "Two-point boundary value problems by the extended Adomian decomposition method," Journal of Computational and Applied Mathematics, vol. 219, no. 1, pp. 253-262, 2008.

[27] S. A. Khuri and A. Sayfy, "A novel approach for the solution of a class of singular boundary value problems arising in physiology," Mathematical and Computer Modelling, vol. 52, no. 3-4, pp. 626-636, 2010.

[28] D. Lesnic, "A computational algebraic investigation of the decomposition method for time-dependent problems," Applied Mathematics and Computation, vol. 119, no. 2-3, pp. 197-206, 2001.

[29] J.-S. Duan and R. Rach, "A new modification of the Adomian decomposition method for solving boundary value problems for higher order nonlinear differential equations," Applied Mathematics and Computation, vol. 218, no. 8, pp. 4090-4118, 2011.

[30] K. Abbaoui and Y. Cherruault, "Convergence of Adomian's method applied to differential equations," Computers \& Mathematics with Applications, vol. 28, no. 5, pp. 103-109, 1994.

[31] Y. Cherruault, "Convergence of Adomian's method," Kybernetes, vol. 18, no. 2, pp. 31-38, 1989.

[32] Y. Cherruault and G. Adomian, "Decomposition methods: a new proof of convergence," Mathematical and Computer Modelling, vol. 18, no. 12, pp. 103-106, 1993.

[33] M. M. Hosseini and H. Nasabzadeh, "On the convergence of Adomian decomposition method," Applied Mathematics and Computation, vol. 182, no. 1, pp. 536-543, 2006.

[34] M. M. Chawla, R. Subramanian, and H. L. Sathi, "A fourth order method for a singular two-point boundary value problem," BIT Numerical Mathematics, vol. 28, no. 1, pp. 88-97, 1988. 


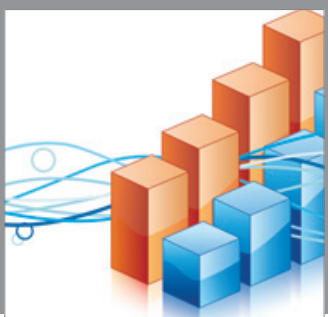

Advances in

Operations Research

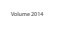

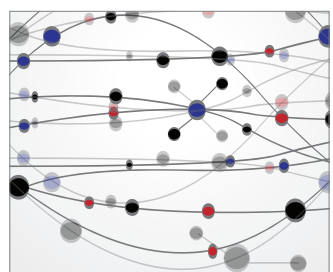

\section{The Scientific} World Journal
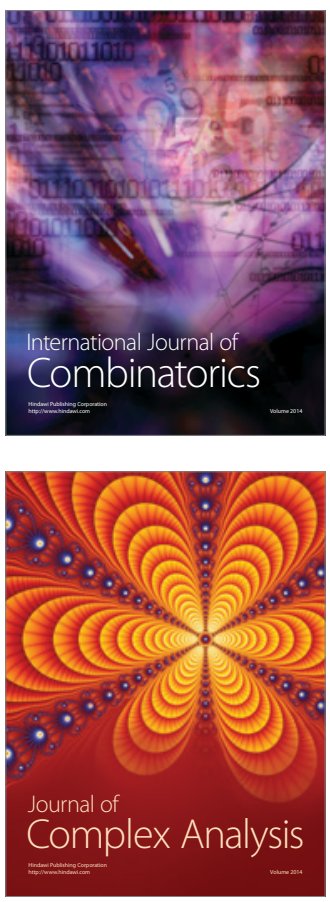

International Journal of

Mathematics and

Mathematical

Sciences
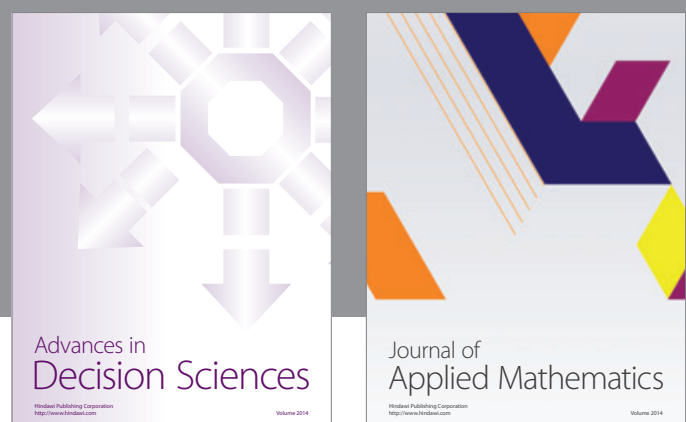

Journal of

Applied Mathematics
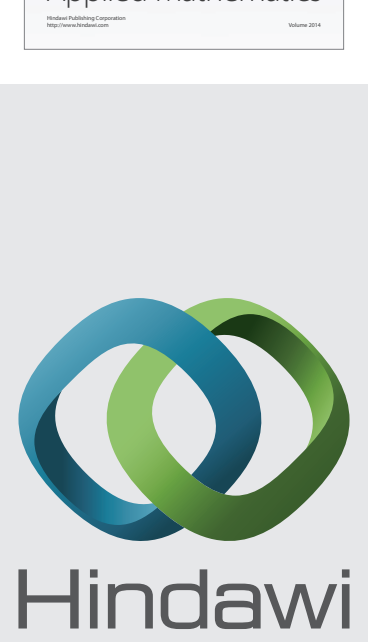

Submit your manuscripts at http://www.hindawi.com
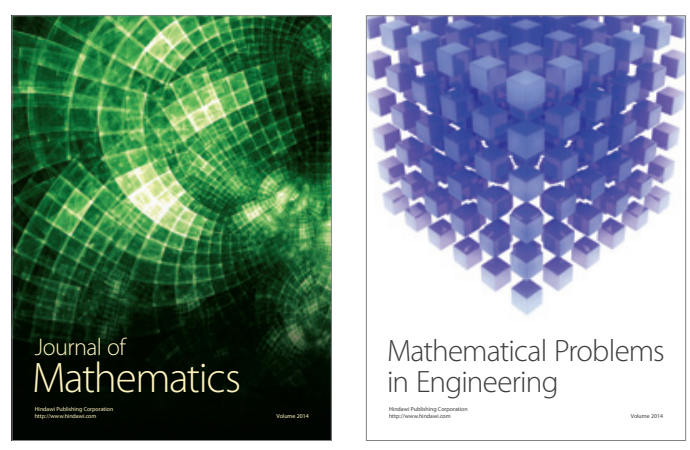

Mathematical Problems in Engineering
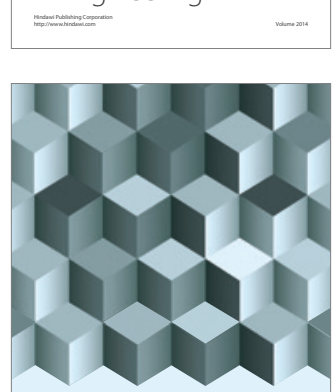

Journal of

Function Spaces
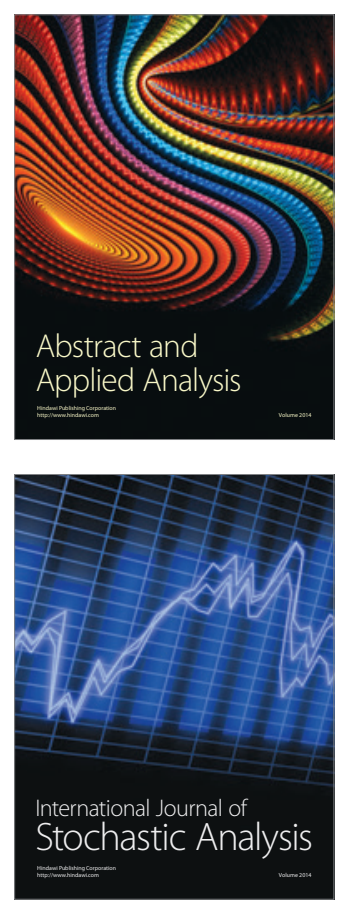

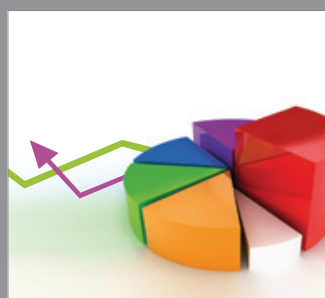

ournal of

Probability and Statistics

Promensencen
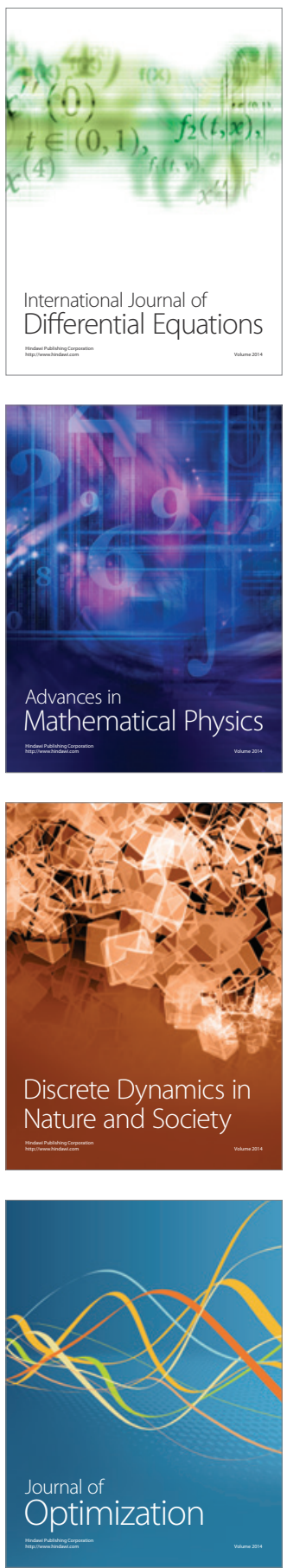